\title{
VARIATION OF PARAMETERS METHOD FOR A THREE-DIMENSIONAL PROBLEM OF CONDENSATION FILM ON AN INCLINED ROTATING DISK
}

\author{
Osman Güngör, Cihat Arslantürk \\ Faculty of Engineering, Ataturk University \\ Erzurum, Turkey \\ osmngngr26@gmail.com,carslan@atauni.edu.tr \\ Received: 28 October 2018; Accepted: 7 March 2019
}

\begin{abstract}
In this paper, the steady three-dimensional problem of condensation film on an inclined rotating disk is considered. The governing nonlinear partial differential equations are reduced to the nonlinear ordinary differential equations system by a similarity transform. The equation system is solved by the variation of parameters method (VPM) which is rather used to solve nonhomogeneous linear differential equations but can also be used to solve nonlinear differential equations. This method has not previously been used to solve a nonlinear condensation problem. The dimensionless velocity and temperature profiles are shown, and the influence of Prandtl number and rotation ratio on the flow field and the Nusselt number are discussed in detail. In order to assess the accuracy of the solutions obtained by this method, the problem is also solved numerically using the Matlab bvp4c solver. The validity of our solutions is verified by the numerical results.
\end{abstract}

MSC 2010: 35A15, 34L30

Keywords: condensation, rotating inclined disk, nonlinear ordinary differential equations, variation of parameters method

\section{Introduction}

The removal of a liquid condensate from a cooled, saturated vapor is important in some engineering applications such as spray cooling, spray coating and chemical vapor accumulation processes commonly used in the semiconductor industry to produce thin films. A complete physical model of the condensation is required for the fluid flow and heat transfer analysis of these processes. A well-known study conducted by Nusselt [1] on condensation on a vertical plate, forms the basis of many theoretical studies of condensation. Koh et al. [2] developed Nusselt's solution taking into account the inertia and convective terms and the vapor resistance in the flow of the condensing fluid. Based on the simplicity of Nusselt's model, Sparrow and Gregg [3] studied the condensation on a disk rotating in steady vapor with a large 
volume. In this study, making use of the well-known study of von Karman [4] on a rotating disk in an infinite fluid environment, they transformed the Navier-Stokes equations into a set of nonlinear ordinary differential equations, and numerically integrated them for a similarity solution for several finite film thicknesses. Their work was extended by adding vapor drag by Beckett et al. [5] and adding suction on the plate by Chary and Sarma [6].

In this study, the flow of liquid film composed of condensing liquid on a disk obliquely positioned under the influence of centrifugal and gravitational forces has been studied. This problem was solved by various researchers using different solution methods. For example, Wang transformed the problem of three-dimensional condensation on an oblique plate into a nonlinear set of ordinary differential equations [7] by using a similarity transformation. Wang solved this problem by the perturbation method for small values of the problem parameter and he solved the problem with the fifth order Runge-Kutta method in order to determine the parameter range in which the perturbation method is valid and presented the results in the literature. The same problem was solved using the Homotopy Analysis Method by Rashidi and Dinarvand [8] using the Homotopy Perturbation Method by Sheikhholeslami et al. [9], using the Differential Transformation Method by Rashidi and Mohimanianpour [10], using the Optimal Homotopy Analysis Method [11], using the Extended Optimal Homotopy Asymptotic Method [12] and using Akbari-Ganji Method [13].

In this study, liquid film formed by the condensing fluid on a rotating disk influenced by both centrifugal force and gravitational force is considered. The problem is expressed by a set of partial differential equations. By the similarity transformation proposed by Wang [7], the problem is transformed into a set of nonlinear ordinary differential equations. This set of equations has been solved using variation of parameters method [14-17] which is rather used to solve nonhomogeneous linear differential equations but also has been proposed by some researchers for the solution of nonlinear differential equations. This method has recently been used by Moore [18] for the first time in a doctoral thesis for non-linear heat transfer problems. Other studies by Moore and Jones [19, 20] have also been included in the literature as the first studies where the method was used in the problems of heat transfer. This study is the first study in which the nonlinear fluid condensation problem was solved by the variation of parameters method.

\section{Mathematical formulation and flow analysis}

In Figure 1, a disk is seen having the angular velocity $\Omega$ rotating in its own plane and in $\beta$ angle horizontally. As a result of the condensation on the disk, a liquid film is formed accumulated with $W$ velocity at $t$ thickness. The mathematical formulation of the problem is stated in the $(x, y, z)$ coordinate system and the $z$ axis being the rotation axis. Since the thickness of the film is too small compared to the radius of the disk, the end effects can be neglected. Moreover, it is stated 
in the literature that the shear stress on the vapor-fluid interface is insignificant [7]. The ambient pressure on the surface of the film is constant at $p_{0}$ and the pressure in the film is only the function of the $z$ coordinate. Assuming that the thermophysical properties of the fluid are constant, the continuity, momentum and energy equations for the steady state conditions are stated in the following form:

$$
\begin{gathered}
u_{x}+u_{y}+u_{z}=0 \\
u u_{x}+v u_{y}+w u_{z}=v\left(u_{x x}+u_{y y}+u_{z z}\right)+\bar{g} \sin \beta \\
u v_{x}+v v_{y}+w v_{z}=v\left(v_{x x}+v_{y y}+v_{z z}\right) \\
u w_{x}+v w_{y}+w w_{z}=v\left(w_{x x}+w_{y y}+w_{z z}\right)-\bar{g} \cos \beta-\frac{p_{z}}{\rho} \\
u T_{x}+v T_{y}+w T_{z}=\alpha\left(T_{x x}+T_{y y}+T_{z z}\right)
\end{gathered}
$$

In the above equations, $u, v$ and $w$ indicate the velocity components in the $x, y$ and $z$ directions, $T$ denotes the temperature, and $\rho, v$ and $\alpha$ denote the density, kinematic viscosity and thermal diffusivity of the fluid, respectively. Assuming zero slip on the disk and zero shear stress on the film surface, the boundary conditions are:

$$
\begin{gathered}
u=-\Omega y, \quad v=\Omega x, \quad w=0, \quad T=T_{w} \quad \text { at } \quad z=0 \\
u_{z}=0, \quad v_{z}=0, \quad w=-W, \quad T=T_{0}, \quad p=p_{0} \quad \text { at } \quad z=t
\end{gathered}
$$

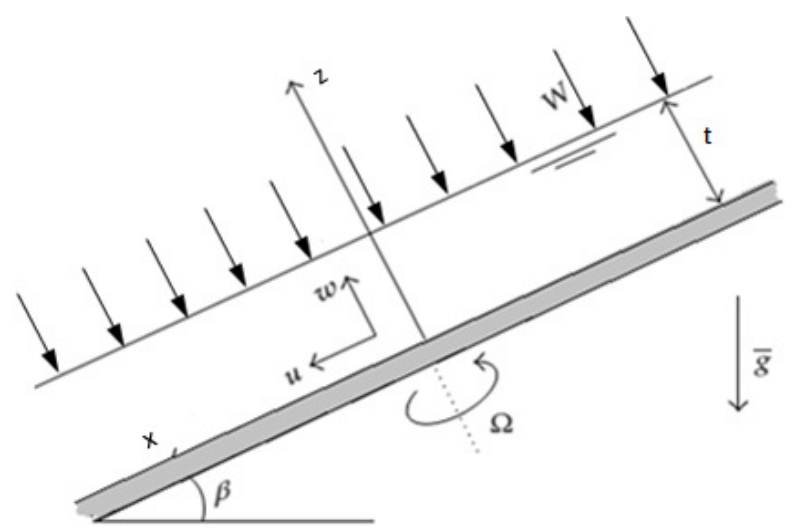

Fig. 1. Schematic diagram of the problem

Wang [7] introduced the following similarity transformation:

$$
\begin{gathered}
u=-\Omega y g(\eta)+\Omega x f^{\prime}(\eta)+\bar{g} \sin \beta k(\eta) / \Omega \\
v=\Omega x g(\eta)+\Omega y f^{\prime}(\eta)+\bar{g} \sin \beta s(\eta) / \Omega
\end{gathered}
$$




$$
w=-2 \sqrt{\Omega v} f(\eta)
$$

where

$$
\eta=z \sqrt{\Omega / \nu}
$$

Continuity Eq. (1) is satisfied automatically with this similarity transformation. Eqs. (2a) and (2b) can be defined in terms of new variables as follows:

$$
\begin{gathered}
f^{\prime \prime \prime}-\left(f^{\prime}\right)^{2}+g^{2}+2 f f^{\prime \prime}=0, \\
g^{\prime \prime}-2 g f^{\prime}+2 f g^{\prime}=0, \\
k^{\prime \prime}-k f^{\prime}+s g+2 f k^{\prime}+1=0, \\
s^{\prime \prime}-g k-s f^{\prime}+2 f s^{\prime}=0 .
\end{gathered}
$$

If the temperature is considered to be the function of $z$ only, the Eq. (3) is

$$
\theta^{\prime \prime}+2 \operatorname{Pr} f \theta^{\prime}=0
$$

where $\operatorname{Pr}=v / \alpha$ is the Prandtl number. The boundary conditions for the new variables are:

$$
\begin{gathered}
f(0)=0, \quad f^{\prime}(0)=0, \quad f^{\prime \prime}(\delta)=0, \\
g(0)=1, \quad g^{\prime}(\delta)=0, \\
k(0)=0, \quad k^{\prime}(\delta)=0, \\
s(0)=0, \quad s^{\prime}(\delta)=0, \\
\theta(0)=0, \quad \theta(\delta)=1,
\end{gathered}
$$

After the flow field is found, desired quantities of the fluid can be calculated. Firstly, the pressure distribution in the film can be obtained by integrating the Eq. (2c):

$$
p(z)=p_{0}+\rho\left\{v\left[\frac{\partial w}{\partial z}-\left.\frac{\partial w}{\partial z}\right|_{z=t}\right]-\frac{1}{2}\left[w^{2}(z)-w^{2}(t)\right]-\bar{g} \cos \beta(z-t)\right\} .
$$

If the net forces on the per unit area in the $x$ and $y$ directions are normalized by, $\bar{g} \rho \sqrt{v / \Omega} \sin \beta$, they are equal to $k^{\prime}(0)$ and $s^{\prime}(0)$ values, respectively. The net flow rates for per unit width in $x$ and $y$ directions are represented by $\int_{0}^{\delta} k(\eta) d \eta$ and $\int_{0}^{\delta} s(\eta) d \eta$ integrals, respectively. 


\section{Variation of parameters method for nonlinear problems}

The method of variation of parameters is generally used to solve nonhomogeneous linear differential equations [21]. However, it is known from the literature that it can also be used to solve nonlinear equations [14-20]. In linear equations, while the term disrupting homogeneity is only the function of the independent variable, in nonlinear equations, this term is also the function of the dependent variable.

Consider the following $n$ order nonlinear differential equation

$$
L \Psi=f\left(X, \Psi, \Psi^{\prime}, \Psi^{\prime \prime}, \cdots, \Psi^{(n-1)}\right)
$$

where $L$ is a linear operator from $n$ order. The homogeneous solution of the Eq. (9) could be expressed as follows:

$$
\Psi_{c}(X)=c_{1} \Psi_{c, 1}(X)+c_{2} \Psi_{c, 2}(X)+\ldots \ldots+c_{n} \Psi_{c, n}(X)
$$

where $c_{1}, c_{2}, \ldots, c_{n}$ are integration constants and $\Psi_{c, 1}, \Psi_{c, 2}$ and $\Psi_{c, n}$ are linear independent solutions of the homogeneous equation. It is desirable that Eq. (9) has a particular solution as follows:

$$
\Psi_{p}(X)=v_{1}(X) \Psi_{c, 1}(X)+v_{2}(X) \Psi_{c, 2}(X)+\cdots+v_{n}(X) \Psi_{c, n}(X)
$$

The solution procedure to be applied for the nonlinear Eq. (9) is same for the linear equations:

$$
\Psi(X)=\Psi_{c}(X)+\Psi_{p}(X)
$$

In Eq. (11), the particular solution functions are found given as:

$$
v_{n}(X)=\int_{0}^{X} \frac{f\left(X, \Psi, \Psi^{\prime}, \cdots, \Psi^{(n-1)}\right) W_{n}(X)}{W\left[\Psi_{c, 1}, \Psi_{c, 2}, \cdots, \Psi_{c, n}\right](X)} d X
$$

In the above equation, $W$ is the Wronskian of independent solutions of the homogeneous equation

$$
W\left[\Psi_{c, 1}, \Psi_{c, 2}, \cdots, \Psi_{c, n}\right]=\left|\begin{array}{cccc}
\Psi_{c, 1} & \Psi_{c, 1} & \ldots & \Psi_{c, n} \\
\Psi_{c, 1}^{\prime} & \Psi_{c, 2}^{\prime} & \ldots & \Psi_{c, n}^{\prime} \\
\vdots & \vdots & \ddots & \vdots \\
\Psi_{c, 1}^{(n-1)} & \Psi_{c, 2}^{(n-1)} & \ldots & \Psi_{c, n}^{(n-1)}
\end{array}\right|
$$

where

$$
W_{n}(X)=(-1)^{n-k} W\left[\Psi_{c, 1}, \cdots \Psi_{c, k-1}, \Psi_{c, k+1}, \cdots \Psi_{c, n}\right](X)
$$


If $f$ in Eq. (13a) depends only on $\Psi$ and $X$, although the method requires an iterative approach and numerical integration, the solution can be considered exact because numerical integration can be performed to an arbitrary degree of precision. If $f$ is also the function of derivatives of $\Psi$, the solution cannot be regarded as an exact solution [18], since these derivatives must be used approximately by using finite difference equations.

\section{Numerical solution by variation of parameters method}

Equations (6) can be defined as follows without taking the highest order derivative term into consideration:

$$
\begin{gathered}
f^{\prime \prime \prime}=F\left(f, g, f^{\prime}, g^{\prime}\right) \\
g^{\prime \prime}=G\left(f, g, f^{\prime}, g^{\prime}\right) \\
k^{\prime \prime}=K\left(f, g, k, s, f^{\prime}, k^{\prime}\right) \\
s^{\prime \prime}=S\left(f, g, k, s, f^{\prime}, s^{\prime}\right) \\
\theta^{\prime \prime}=\Theta\left(f, \theta^{\prime}, \operatorname{Pr}\right)
\end{gathered}
$$

In the above equations, the functions $F, G, K, S$, and $\Theta$ become:

$$
\begin{gathered}
F=\left(f^{\prime}\right)^{2}-g^{2}-2 f f^{\prime \prime} \\
G=2 g f^{\prime}-2 f g^{\prime} \\
K=k f^{\prime}-s g-2 f k^{\prime}-1 \\
S=g k+s f^{\prime}-2 f s^{\prime} \\
\Theta=-2 \operatorname{Pr} f \theta^{\prime}
\end{gathered}
$$

Equations (14) are transformed into equations similar to Eq. (12) according to the variation of parameters method described in the previous section. Here, this procedure will only be explained for the Eq. (14a), and only results will be presented for other equations.

The homogeneous solution of the Eqs. (14a) are

$$
f_{c}(\eta)=c_{1} f_{c, 1}(\eta)+c_{2} f_{c, 2}(\eta)+c_{3} f_{c, 3}(\eta)=c_{1} \eta^{2}+c_{2} \eta+c_{3}
$$

where $c_{1}, c_{2}$ and $c_{3}$ are integration constants and $f_{c, 1}, f_{c, 2}$ and $f_{c, 3}$ are linear independent solutions of the above homogeneous equation. It is desirable that Eq. (14a) has a particular solution as follows: 


$$
f_{p}(\eta)=\eta^{2} v_{1}(\eta)+\eta v_{2}(\eta)+v_{3}(\eta)
$$

The solution of the nonlinear Eq. (14a) is the sum of homogeneous and particular solutions.

$$
f(\eta)=c_{1} \eta^{2}+c_{2} \eta+c_{3}+\eta^{2} v_{1}(\eta)+\eta v_{2}(\eta)+v_{3}(\eta)
$$

Equations (13a)-(13c) and the particular solution functions are given by

$$
\begin{aligned}
& v_{1}(\eta)=\frac{1}{2} \int_{0}^{\eta} F\left(f, g, f^{\prime}, g^{\prime}\right) d \eta \\
& v_{2}(\eta)=-\int_{0}^{\eta} \not F\left(f, g, f^{\prime}, g^{\prime}\right) d \eta \\
& v_{3}(\eta)=\frac{1}{2} \int_{0}^{\eta} \eta^{2} F\left(f, g, f^{\prime}, g^{\prime}\right) d \eta
\end{aligned}
$$

Therefore,

$$
f(\eta)=-\frac{1}{2} \eta^{2} \int_{0}^{\delta} F\left(f, g, f^{\prime}, g^{\prime}\right) d \eta+\eta^{2} v_{1}(\eta)+\eta v_{2}(\eta)+v_{3}(\eta)
$$

using the boundary conditions given by $(7 \mathrm{a}), c_{1}, c_{2}$ and $c_{3}$ constants are as follows:

$$
c_{1}=-\frac{1}{2} \int_{0}^{\delta} F\left(f, g, f^{\prime}, g^{\prime}\right) d \eta, \quad c_{2}=0, \quad c_{3}=0
$$

$f$ function is obtained by substituting the functions in Eqs.(19) and $c_{1}, c_{2}$ and $c_{3}$ constants in Eq. (21) into Eq. (20).

$$
\begin{aligned}
f(\eta) & =-\frac{\eta^{2}}{2} \int_{0}^{\delta} F\left(f, g, f^{\prime}, g^{\prime}\right) d \eta+\frac{\eta^{2}}{2} \int_{0}^{\eta} F\left(f, g, f^{\prime}, g^{\prime}\right) d \eta \\
& -\eta \int_{0}^{\eta} \not F\left(f, g, f^{\prime}, g^{\prime}\right) d \eta+\frac{1}{2} \int_{0}^{\eta} \eta^{2} F\left(f, g, f^{\prime}, g^{\prime}\right) d \eta
\end{aligned}
$$

Because the $f$ appears on both sides and $g$ the right side of the Eq. (22a), an iterative approach is required for the solution. An initial guess for the $f$ and $g$ functions plugged into the right side of the Eq. (22a)

$$
\begin{aligned}
f_{n+1}= & -\frac{\eta^{2}}{2} \int_{0}^{\delta} F\left(f_{n}, g_{n}, f_{n}^{\prime}, g_{n}^{\prime}\right) d \eta+\frac{\eta^{2}}{2} \int_{0}^{\eta} F\left(f_{n}, g_{n}, f_{n}^{\prime}, g_{n}^{\prime}\right) d \eta \\
& -\eta \int_{0}^{\eta} \psi F\left(f_{n}, g_{n}, f_{n}^{\prime}, g_{n}^{\prime}\right) d \eta+\frac{1}{2} \int_{0}^{\eta} \eta^{2} F\left(f_{n}, g_{n}, f_{n}^{\prime}, g_{n}^{\prime}\right) d \eta
\end{aligned}
$$


Here, $f_{n}$ is the $n$th approximation or iteration of $f$ and $f_{n+1}$ is the next or $n+1$ iteration of $f$ and $n \geq 0$. For the numerical solution of the integrals in the Eqs. (22b), the problem region $(0, \delta)$ is divided into $M$ sub-region. Assigning an initial guess vector for each of the functions, the right sides of the equations are computed by the trapezoidal rule. The derivatives in Eq. (22b) are used approximately by using finite difference equations. The relative approximate error between the vectors representing the new values of the functions and the guess vectors is computed and when the error reaches the desired tolerance value, the iteration is terminated. As can be seen from Eqs. (22a), the variation of parameters method reduces the solution of a nonlinear set of differential equations to numerical integration solution.

The same procedure is applied to Eqs. (14b)-(14e) and the other functions are obtained as follows:

$$
\begin{gathered}
g(\eta)=1-\eta \int_{0}^{\delta} G d \eta+\eta \int_{0}^{\eta} G d \eta-\int_{0}^{\eta} \eta G d \eta \\
k(\eta)=-\eta \int_{0}^{\delta} K d \eta+\eta \int_{0}^{\eta} K d \eta-\int_{0}^{\eta} \eta K d \eta \\
s(\eta)=-\eta \int_{0}^{\delta} S d \eta+\eta \int_{0}^{\eta} S d \eta-\int_{0}^{\eta} \eta S d \eta \\
\theta(\eta)=\frac{\eta}{\delta}\left[1-\delta \int_{0}^{\delta} \Theta d \eta-\int_{0}^{\delta} \eta \Theta d \eta\right]+\eta \int_{0}^{\eta} \Theta d \eta-\int_{0}^{\eta} \eta \Theta d \eta
\end{gathered}
$$

\section{Results and discussion}

In this part of the paper, firstly the convergence and accuracy of the solution obtained by VPM is investigated. The solution of the nonlinear ordinary differential equation set given by (22a)-(22e) is obtained iteratively. The integrals in these equations have been solved numerically with the trapezoidal rule by dividing the problem region into the $M$ sub-region. The minimum number of sub-regions required for a solution independent of the number of sub-regions has been identified by examining Table 1 . In Table 1 , it is seen that values of $f, g, k$ and $s$ functions on surface of the film and the derivative values of these functions on the surface of disk change depending on the number of sub-regions used in the solution. For all cases from Table 1 , it is seen that the value of $M=200$ is sufficient to obtain a solution independent of the number of sub-regions. However, for more reliable solutions, numerical integrals have been calculated with $M=500$ sub-region number in all solutions. 
Table 1. Effect of the number of sub-region on the convergence of the solution for $\delta=1.0$

\begin{tabular}{|c|c|c|c|c|c|c|c|c|}
\hline$M$ & $f(\delta)$ & $g(\delta)$ & $k(\delta)$ & $-s(\delta)$ & $f^{\prime \prime}(0)$ & $-g^{\prime}(0)$ & $k^{\prime}(0)$ & $-s^{\prime}(0)$ \\
\hline 10 & 0.2131070 & 0.7756361 & 0.4257919 & 0.1360428 & 0.7000229 & 0.3761105 & 0.8896359 & 0.2302441 \\
\hline 20 & 0.2134697 & 0.7751300 & 0.4261382 & 0.1359606 & 0.7040528 & 0.3743158 & 0.8904792 & 0.2279164 \\
\hline 50 & 0.2135597 & 0.7749763 & 0.4262278 & 0.1359318 & 0.7052161 & 0.3737458 & 0.8907142 & 0.2272071 \\
\hline 100 & 0.2135717 & 0.7749535 & 0.4262400 & 0.1359272 & 0.7053842 & 0.3736589 & 0.8907477 & 0.2271013 \\
\hline 150 & 0.2135739 & 0.7749492 & 0.4262422 & 0.1359264 & 0.7054154 & 0.3736425 & 0.8907539 & 0.2270814 \\
\hline 200 & 0.2135746 & 0.7749477 & 0.4262430 & 0.1359260 & 0.7054263 & 0.3736367 & 0.8907561 & 0.2270744 \\
\hline 250 & 0.2135750 & 0.7749470 & 0.4262433 & 0.1359259 & 0.7054314 & 0.3736340 & 0.8907571 & 0.2270711 \\
\hline 300 & 0.2135752 & 0.7749466 & 0.4262435 & 0.1359258 & 0.7054342 & 0.3736325 & 0.8907576 & 0.2270694 \\
\hline 350 & 0.2135753 & 0.7749464 & 0.4262436 & 0.1359258 & 0.7054358 & 0.3736317 & 0.8907579 & 0.2270683 \\
\hline 400 & 0.2135754 & 0.7749462 & 0.4262437 & 0.1359257 & 0.7054369 & 0.3736311 & 0.8907581 & 0.2270676 \\
\hline 450 & 0.2135754 & 0.7749461 & 0.4262438 & 0.1359257 & 0.7054376 & 0.3736307 & 0.8907583 & 0.2270671 \\
\hline 500 & 0.2135754 & 0.7749460 & 0.4262438 & 0.1359257 & 0.7054382 & 0.3736304 & 0.8907584 & 0.2270668 \\
\hline
\end{tabular}

Figure 2 shows how the dimensionless temperature distribution converges from the initial guess to the solution. In this example, $M=500$ was taken and convergence was achieved in 136 iterations.

In order to investigate the accuracy of the VPM solution, a nonlinear set of differential equations was also solved numerically by the Matlab "bvp4c" solver. The numerical solution (NUM) and VPM solutions are represented in Table 2. The table shows that there is an excellent agreement between the results.

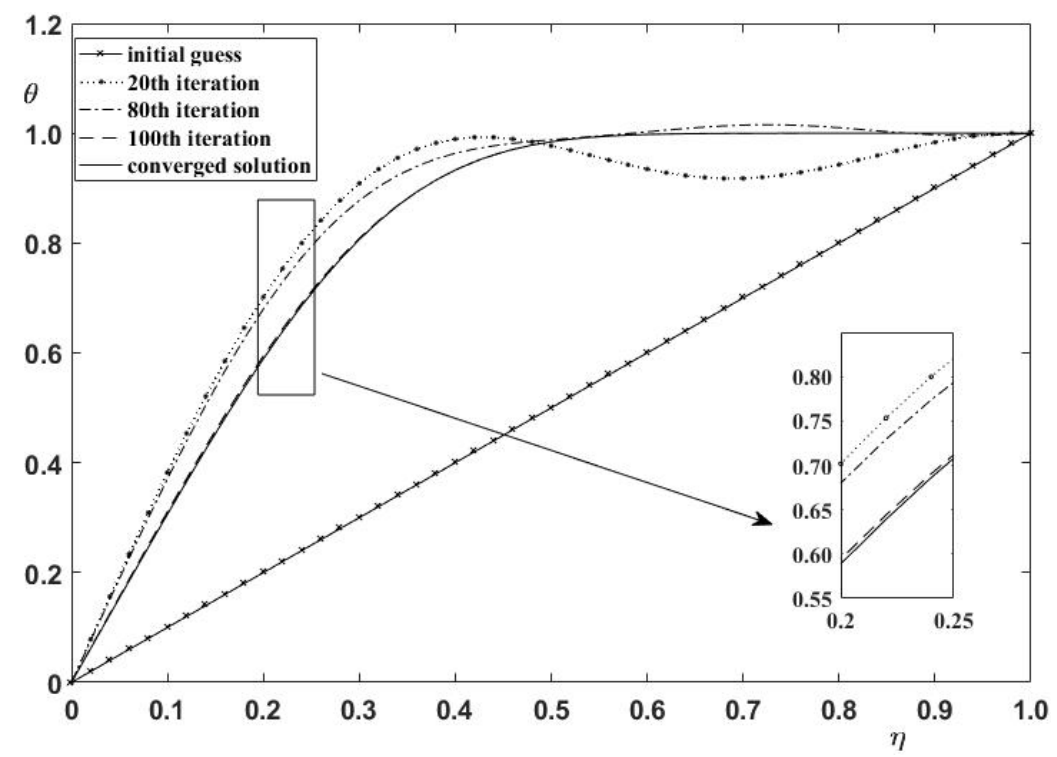

Fig. 2. Convergence of non-dimensional temperature profile $(\operatorname{Pr}=100, \delta=1.0)$ 
The results obtained by Rashidi and Dinarvand [8] with the Homotopy Analysis Method (HAM), the results obtained by Rashidi and Mohimanianpour [10] with the Differential Transform Method (DTM), the results by Hassan and Rashidi [11] and VPM results and numerical results are compared in Table 3. In the Table, it is observed that particularly the results of OHAM, DTM, VPM and numerical solution are in consistence.

Table 2. Comparison of VPM and numerical solution for $f, g, k$ and $s$ functions for $\delta=0.5$

\begin{tabular}{|c|c|c|c|c|c|c|c|c|}
\hline \multirow{2}{*}{$\eta$} & \multicolumn{2}{|c|}{$f$} & \multicolumn{2}{c|}{$g$} & \multicolumn{2}{c|}{$k$} & \multicolumn{2}{c|}{$-s$} \\
\cline { 2 - 9 } & VPM & NUM & VPM & NUM & VPM & NUM & VPM & NUM \\
\hline 0 & 0.000000 & 0.000000 & 1.000000 & 1.000000 & 0.000000 & 0.000000 & 0.000000 & 0.000000 \\
\hline 0.1 & 0.002247 & 0.002247 & 0.992286 & 0.992286 & 0.044418 & 0.044418 & 0.003932 & 0.003932 \\
\hline 0.2 & 0.008328 & 0.008328 & 0.985418 & 0.985417 & 0.078877 & 0.078877 & 0.007431 & 0.007432 \\
\hline 0.3 & 0.017266 & 0.017266 & 0.980055 & 0.980054 & 0.103421 & 0.103421 & 0.010163 & 0.010163 \\
\hline 0.4 & 0.028098 & 0.028098 & 0.976660 & 0.976659 & 0.118102 & 0.118102 & 0.011891 & 0.011891 \\
\hline 0.5 & 0.039873 & 0.039873 & 0.975501 & 0.975500 & 0.122980 & 0.122980 & 0.012481 & 0.012481 \\
\hline
\end{tabular}

Table 3. Comparison of the results of VPM with other methods in the literature for $\delta=1.0$

\begin{tabular}{|c|c|c|c|c|c|c|}
\hline & $\eta$ & HAM [8] & OHAM [18] & DTM [10] & VPM & NUM \\
\hline \multirow{5}{*}{$f(\eta)$} & 0.2 & 0.012675 & 0.012800 & 0.012824 & 0.012824 & 0.012824 \\
\hline & 0.4 & 0.045894 & 0.046281 & 0.046535 & 0.046535 & 0.046535 \\
\hline & 0.6 & 0.093341 & 0.094217 & 0.094762 & 0.094763 & 0.094762 \\
\hline & 0.8 & 0.149674 & 0.151124 & 0.152032 & 0.152032 & 0.152032 \\
\hline & 1.0 & 0.210237 & 0.212274 & 0.213575 & 0.213575 & 0.213575 \\
\hline \multirow{5}{*}{$g(\eta)$} & 0.2 & 0.916605 & - & 0.926994 & 0.926994 & 0.926994 \\
\hline & 0.4 & 0.843679 & - & 0.863125 & 0.863125 & 0.863125 \\
\hline & 0.6 & 0.788272 & - & 0.814575 & 0.814575 & 0.814575 \\
\hline & 0.8 & 0.754295 & - & 0.784796 & 0.784796 & 0.784795 \\
\hline & 1.0 & 0.743051 & - & 0.774945 & 0.774945 & 0.774944 \\
\hline \multirow{5}{*}{$k(\eta)$} & 0.2 & 0.168154 & 0.159275 & 0.158444 & 0.158444 & 0.158444 \\
\hline & 0.4 & 0.296762 & 0.280230 & 0.278584 & 0.278584 & 0.278584 \\
\hline & 0.6 & 0.386586 & 0.364424 & 0.362047 & 0.362047 & 0.362047 \\
\hline & 0.8 & 0.438927 & 0.413499 & 0.410583 & 0.410583 & 0.410583 \\
\hline & 1.0 & 0.455797 & 0.429372 & 0.426244 & 0.426243 & 0.426243 \\
\hline \multirow{5}{*}{$-s(\eta)$} & 0.2 & 0.037467 & 0.044681 & 0.044332 & 0.044332 & 0.044332 \\
\hline & 0.4 & 0.070290 & 0.083628 & 0.082971 & 0.082971 & 0.082971 \\
\hline & 0.6 & 0.112194 & 0.113081 & 0.112194 & 0.112194 & 0.112194 \\
\hline & 0.8 & 0.130038 & 0.131057 & 0.130038 & 0.130038 & 0.130038 \\
\hline & 1.0 & 0.135926 & 0.136982 & 0.135926 & 0.135925 & 0.135925 \\
\hline
\end{tabular}


Table 4 shows the effect of the Prandtl number on the non-dimensional temperature distributions. From the table, it shows that the VPM agree excellently with numerical solutions and Akbari_Ganji Method [13].

Table 4. Comparison of the non-dimensional temperature distributions obtained by AGM, VPM and numerical solution when $\delta=0.5$

\begin{tabular}{|c|c|c|c|c|c|c|c|}
\hline \multicolumn{4}{|c|}{$\operatorname{Pr}=0.700$} & \multicolumn{2}{c|}{$\operatorname{Pr}=5.00$} & \multicolumn{2}{c|}{$\operatorname{Pr}=100$} \\
\hline$\eta$ & AGM [13] & VPM & NUM & VPM & NUM & VPM & NUM \\
\hline 0.0 & 0.000000 & 0.000000 & 0.000000 & 0.000000 & 0.000000 & 0.000000 & 0.000000 \\
\hline 0.1 & 0.200554 & 0.200554 & 0.200554 & 0.203951 & 0.203951 & 0.273954 & 0.273954 \\
\hline 0.2 & 0.401036 & 0.401036 & 0.401036 & 0.407379 & 0.407379 & 0.534310 & 0.534310 \\
\hline 0.3 & 0.601278 & 0.601278 & 0.601278 & 0.609074 & 0.609074 & 0.754161 & 0.754160 \\
\hline 0.4 & 0.801032 & 0.801032 & 0.801032 & 0.807288 & 0.807288 & 0.910224 & 0.910224 \\
\hline 0.5 & 1.000000 & 1.000000 & 1.000000 & 1.000000 & 1.000000 & 1.000000 & 1.000000 \\
\hline
\end{tabular}

After proving the accuracy and convergence of the solution obtained with VPM, the flow of condensing fluid and the variation of the heat transfer with some parameters will be investigated.

In Figure 3, the change of non-dimensional radial velocity profiles versus similarity parameter is shown. In Figure 4, the change of $k^{\prime}(0)$ and $-s^{\prime}(0)$, representing the non-dimensional shear stresses in the $x$ and $y$ directions; with dimensionless film thickness is shown. It can be seen from Figure 3 that $k^{\prime}(0)$ increases linearly as thickness increases, and the increase is also larger than $-s^{\prime}(0)$.

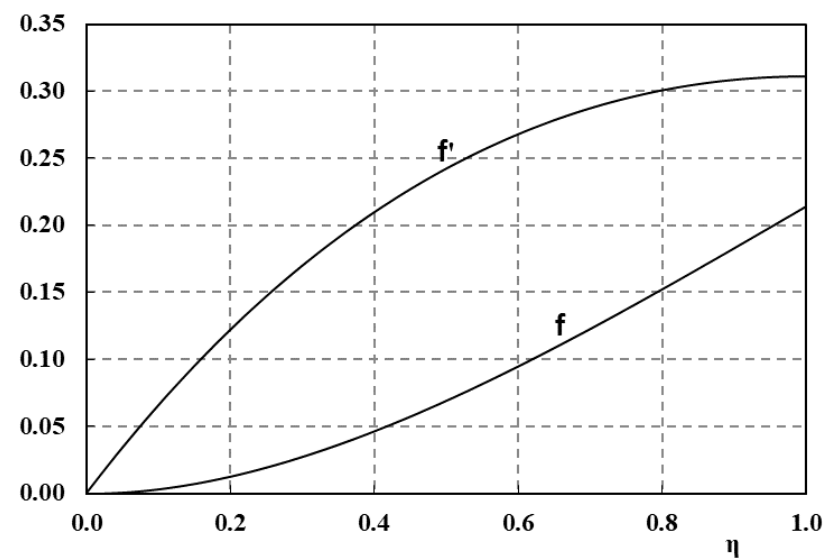

Fig. 3. The change of non-dimensional radial velocity profiles and similarity parameter for $\delta=1.0$

In Figure $5, f^{\prime \prime}(0)$ and $-g^{\prime}(0)$ are expressed as a function of film thickness. The function $f^{\prime \prime}(0)$, which has the same values as the results given by Wang [7], passes from the maximum value of 0.7085 in $\delta=1.08$ value and is asymptote to the value 
of 0.51023 . The function $g^{\prime}(0)$ passes from a minimum in $\delta=2.82$ value and is asymptote to the value of 0.6159 .

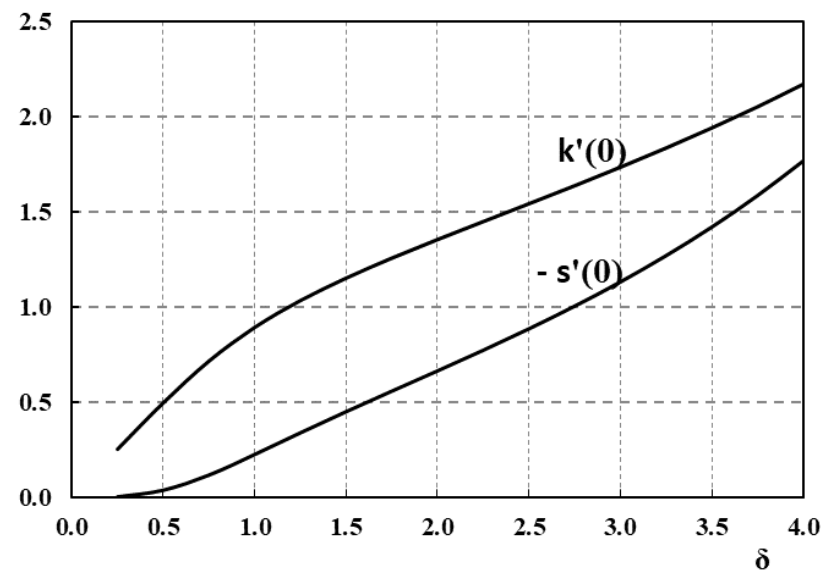

Fig. 4. As a function of the dimensionless film thickness, $k^{\prime}(0)$ and $-s^{\prime}(0)$ being the dimensionless shear stresses in the $x$ and $y$ directions

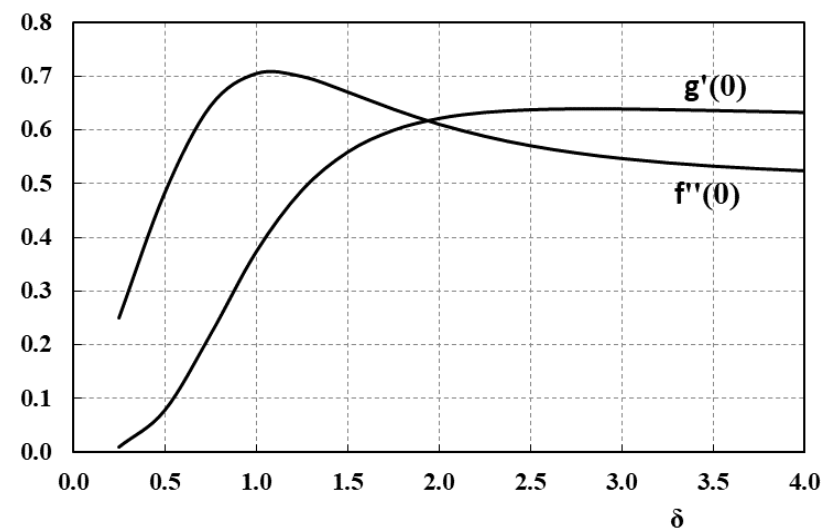

Fig. 5. $f^{\prime \prime}(0)$ and $-g^{\prime}(0)$ initial values for the rotating flow

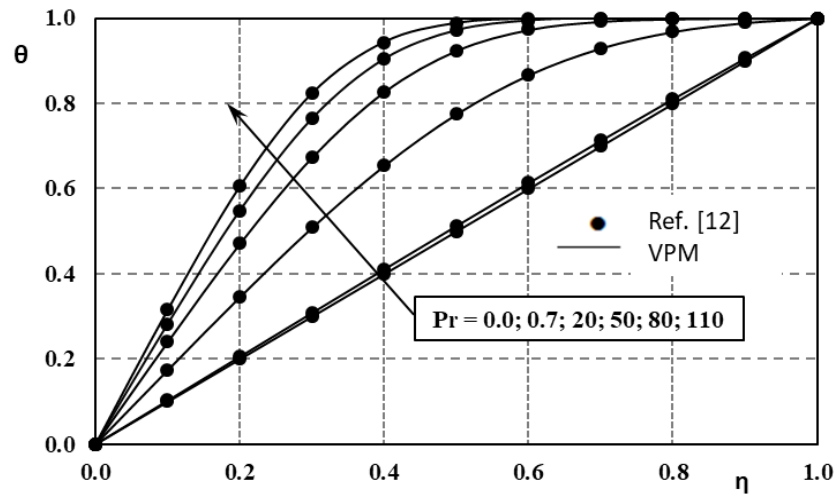

Fig. 6 . The change of normalized temperature profiles with similarity parameter for $\delta=1.0$ 


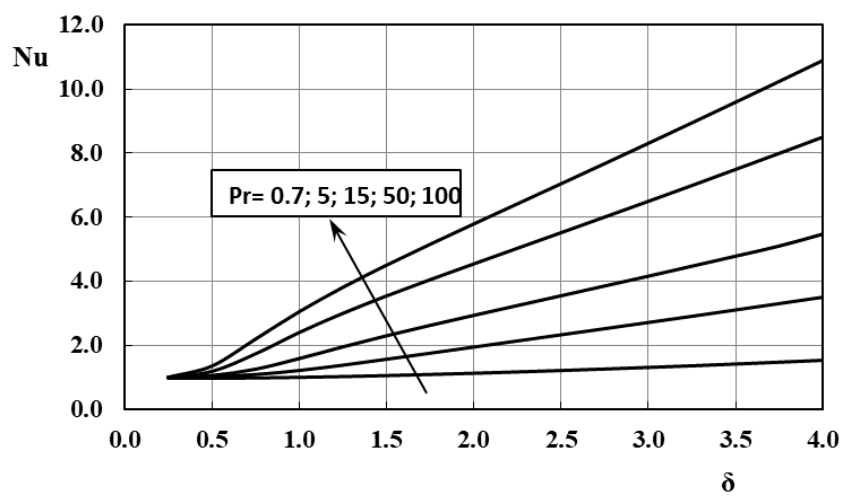

Fig. 7. The change of Nusselt number with dimensionless film thickness for various Prandtl numbers

The normalized temperature profile for different Prandtl numbers are illustrated in Figure 6. The value of Prandtl number ranges from about zero for liquid metals to 0.7 for water, and 110 for some specific liquid metals. Figure 7 shows the change in the Nusselt number with dimensionless film thickness. Although the effect of the Nusselt number is not sensible in small Prandtl numbers, it increases with the increase in thickness; ultimately, in thicknesses of about zero, it reaches a maximum of 1 .

\section{Conclusions}

In this paper, a three-dimensional condensation problem on an inclined rotating disk was taken into consideration. The problem, expressed as a set of partial differential equations, was transformed into a set of a nonlinear ordinary differential equations via a similarity transformation given by Wang [7]. This set of ordinary differential equation is solved with the variation of parameters method (VPM) which is mostly used to solve nonhomogeneous linear differential equations but can also be used to solve nonlinear differential equations. This is the first study where a nonlinear condensation problem of VPM was used to solve the mathematical model. In order to study the validity of VPM solutions, the problem was also solved numerically with the MATLAB bvp4c solver. The comparisons showed that VPM solutions were completely consistent with the results of OHAM, AGM, HPM, DTM and the numerical solution.

\section{References}

[1] Nusselt, W. (1916). Die Oberffachenkondensation des Wasserdampfes. Z Vereines Deutsch Ing., 60, 541-546, 569-575.

[2] Koh, J.C.Y., Sparrow, E.M., \& Hartnett, J.P. (1961). The two phase boundary layer in laminar film condensation. Int. J. Heat and Mass Transf., 2, 69-82. 
[3] Sparrow, E,M., \& Gregg, J.L. (1959). A boundary layer treatment of laminer film condensation. J. Heat Transfer, 81, 13-18.

[4] Von Karman, T. (1921). Uber laminare und turbulente reibung. Zeitschrift fur Angewandte Mathematik und Mechanik, 1, 233-52.

[5] Beckett, P.M., Hudson, P.C., \& Poots, G. (1963). Laminar film condensation due to a rotating disk. Journal of Engineering Mathematics, 7, 63-73.

[6] Chary, S.P., \& Sarma, P.K. (1976). Condensation on a rotating disk with constant axial suction. Journal of Heat Transfer, 98, 682-84.

[7] Wang, C.Y. (2007). Condensation film on an inclined rotating disk. App. Math. Modelling, 31, 1582-1593.

[8] Rashidi, M.M., \& Dinarvand, S. (2009). Purely analytic approximate solutions for steady threedimensional problem of condensation film on inclined rotating disk by homotopy analysis method. Nonlinear Analysis:Real World Applications, 10, 2346-2356.

[9] Sheikholeslami, M., Ashorynejad, H.R., Ganji, D.D., \& Yıldırım, A. (2012). Homotopy perturbation method for three-dimensional problem of condensation film on inclined rotating disk. Scientia Iranica B, 19, 437-442.

[10] Rashidi, M.M., \& Mohimanianpour, S.A. (2010). Analytic solution of steady three-dimensional problem of condensation film on inclined rotating disk by differential transform method. Mathematical Problems in Engineering, 1-15.

[11] Hassan H.N., \& Rashidi, M.M., (2013). Analytical solution for three-dimensional steady flow of condensation film on inclined rotating disk by optimal homotopy analysis method. Walailak Journal of Science and Technology, 10, 479-498.

[12] Ullah H., Islami S., \& Fiza M., (2016) Analytical solution for three-dimensional problem of condensation film on inclined rotating disk by extended asymptotic optimal homotopy method. Iran J. Sci. Technol. Trans. Mech. Eng. 40, 265-273.

[13] Berkan, S., Hoseini, S.R., \& Ganji D.D. (2017) Analytical investigation of steady three dimensional problem of condensation film on inclined rotating disk by Akbari-Ganji's method. Propulsion and Power Research, 6, 277-284.

[14] Mohyud-Din, S.T., Noor, M.A., \& Waheed, A. (2010). Variation of parameters method for initial and boundary value problems. World Applied Sciences Journal, 11, 622-39.

[15] Rahmatullah., \& Mohyud-Din, S.T. (2013). Variation of parameters method for nonlinear diffusion equations. International Journal of Modern Applied Physics, 3, 48-56.

[16] Keckic, J. (1976). Additions to Kamkes treatise VII: variation of parameters for nonlinear second order differential equations. Publikacije Elektrotehnickog fakulteta, Serija Matematika i fizika, 544, 31-36.

[17] Bogdanova, M.P. (1962). On a generalization of the method of variation of parameters. Doklady Akademii nauk BSSR, 6, 285-87.

[18] Moore, T.J. (2014). Application of variation of parameters to solve nonlinear multimode heat transfer problems. Ph. D Thesis, Brigham Young University.

[19] Moore, T.J., \& Jones, M.R. (2014). Analysis of the conduction-radiation problem in absorbing, emitting, non-gray planar media using an exact method. Int. J. Heat and Mass Transf., 73, 804-809.

[20] Moore, T.J., \& Jones, M.R. (2015). Solving nonlinear heat transfer problems using variation of parameters method. Int. J. Therm. Sci., 93, 29-35.

[21] Dawkins, P. (2011). Differential Equations (Vol. 503). Lamar University Press. 